\title{
EFEITOS DA IMPLEMENTAÇÃO DE HISTÓRIAS DIGITAIS NA PRODUÇÃO ORAL DE APRENDIZES DE INGLÊS: UM ESTUDO EMBASADO EM TAREFAS
}

\author{
Juliane Regina TREVISOL* \\ Raquel Carolina Ferraz D’ELY**
}

- RESUMO: Devido à rapidez em que as mudanças tecnológicas se dão no mundo contemporâneo e às possibilidades que as novas ferramentas trazem para a construção de conhecimentos em contextos variados, é crescente o interesse de pesquisadores e educadores acerca da inserção de tecnologias digitais aliadas à educação. Porém, muitos são ainda os questionamentos sobre quão eficiente para a aprendizagem seria a implementação de atividades com tecnologias digitais, em especial em contextos de segunda língua (L2). Assim, esta pesquisa investigou os efeitos de um ciclo de tarefas (ELLIS, 2003) com histórias digitais na produção oral em L2 de aprendizes de inglês em uma universidade pública da Bahia. Dados de produção oral de quatorze participantes foram coletados em três momentos - pré e pós-testes - via Whatsapp e analisados considerando-se sua complexidade, acurácia, fluência e densidade lexical (SKEHAN, 2003). Os resultados, em geral, evidenciam uma melhora na fala dos participantes para algumas das medidas investigadas, mesmo em um curto espaço de tempo. Por fim, discute-se o potencial da história digital como ferramenta para o aprimoramento da L2 em contextos formais de ensinoaprendizagem, considerando-se a necessidade de mais pesquisas que busquem compreender o uso de tecnologias na sala de aula de línguas.

- PALAVRAS-CHAVE: Tecnologia. Histórias Digitais. Tarefas. Produção Oral. Aprendizagemensino de Línguas. Sala de aula.

\section{Introdução}

Sabe-se que, de modo geral, todo aprendiz de segunda língua ${ }^{1}$ (L2) quer ser capaz de usar a língua com fluência, sem erros e de forma apropriada em diversas situações reais de uso (ELLIS, 2003). Porém, tal tarefa tende a ser um tanto quanto árdua, ao

\footnotetext{
* Universidade do Estado da Bahia (UNEB). Jacobina - BA - Brasil. jtrevisol@uneb.br. ORCID: 0000-0001-6847-2221.

*** Universidade Federal de Santa Catarina (UFSC). Florianópolis - SC - Brasil. raqueldely@gmail.com. ORCID: 000-0003-2851-1868.

1 Neste artigo, o termo segunda língua é usado como sinônimo de língua estrangeira (LE), apesar das distinções conhecidas entre os dois conceitos.
} 
contrário do que muitos outdoors e propagandas de cursos de idioma propõem, por exemplo. Em contextos de aprendizagem-ensino de L2, muito esforço tende a ser feito pelo professor a fim de dividir o tempo e proporcionar oportunidades suficientes para o desenvolvimento de várias competências da língua-alvo, de modo minimamente autônomo. Com relação à produção oral em L2 em especial, o pouco tempo disponível em sala de aula tem sido um dos grandes obstáculos para que tal habilidade possa ser desenvolvida adequadamente (APPEL; BORGES, 2011; WEISSHEIMER; CALDAS; MARQUES, 2018). Aliada à escassez de tempo, pouca atenção parece ser dada pelo professor a tal questão, possivelmente devido às complexidades inerentes à evolução da própria habilidade (WEISSHEIMER, 2007), dentre outros fatores. Portanto, o tempo na sala de aula tende a ser insuficiente e muito há que ser feito para que a L2 se desenvolva integralmente, sobretudo quando se busca um grau mais elevado de proficiência linguística.

Deste modo, oportunidades adicionais de produção oral em L2 se fazem indispensáveis a fim de se fomentar seu desenvolvimento. Isso porque, seguindo uma perspectiva de processamento cognitivo da linguagem, a fala é entendida como uma competência extremamente complexa (LEVELT, 1989), a qual demanda, portanto, atenção e prática para ser desenvolvida. Weissheimer, Caldas e Marques (2018) sugerem, por exemplo, o uso de tarefas orais por meio de whatsapp a fim de promover momentos de prática e fortalecimento da oralidade em L2 na sala de aula. Neste estudo, com 27 alunos de inglês (nível básico) de uma escola privada, percebeu-se melhora no desempenho oral dos aprendizes, em termos de precisão gramatical (ou acurácia ${ }^{2}$ ), após dois meses de atividades e um total de 4 tarefas gravadas em áudio. Desse modo, estudos como este servem de incentivo a outras propostas pensadas para a aprendizagem de línguas, já que fomentam a prática e uso efetivo da L2, especialmente integrando tarefas pedagógicas e tecnologias digitais ${ }^{3}$, cada vez mais presentes nas nossas atividades diárias.

A tecnologia, de modo geral, parece estar hoje em todo lugar, integrando nossos afazeres cotidianos nos mais diversos ambientes e para as mais diversas finalidades de uso (i.e., Smartphones, email, Moodle, Whatsapp, Instagram, Skype, YouTube, Spotify, dentre inúmeros outros exemplos). Não há dúvida quanto à sua presença cada vez mais constante na nossa rotina, seja ela de lazer, de trabalho ou de estudo. Considerando-se o contexto educacional, por exemplo, o impacto de certas ferramentas da Web 2.0 blogs, wikis, sites de mídia social, sites de criação e compartilhamento de vídeo, dentre outros - tem sido evidenciado por pesquisas como revolucionário, devido à crescente quantidade de educadores e aprendizes que tem começado a usar tais recursos (WANG; VÁSQUEZ, 2012).

Acurácia, entendida como habilidade de evitar erros, foi medida pelo número de erros por 100 palavras (WEISSHEIMER; CALDAS; MARQUES, 2018).

3 Tecnologia digital é aqui entendida como sinônimo de tecnologia de informação e comunicação (TIC), seguindo Evans (2009). Além disso, está também relacionada ao termo 'mídia digital', que se refere, por exemplo, há elementos - como a internet, telefones celulares, jogos de computador, televisão interativa, dentre outros (BUCKINGHAM, 2007). 
No âmbito de ensino e aprendizagem de L2, de modo especial, entende-se que as tecnologias digitais têm permitido a muitos aprendizes experimentar o processo de aprendizagem de modo diferente, mais interativo e criativo. $\mathrm{O}$ uso de certos recursos de comunicação (i.e., chats, fóruns, videoconferências, dentre outros) amplia a possibilidade de se interligarem as quatro habilidades-base da L2 (leitura, escrita, fala, e compreensão auditiva), o que possibilita seu melhor desenvolvimento; além disso, ampliam-se as chances de que o uso da L2 seja, assim, mais autêntico e significativo (TUMOLO, 2006, 2015). Porém, muitos ainda são os questionamentos acerca de como incorporar essas novas tecnologias com tarefas para o desenvolvimento de L2 (GONZÁLEZ-LLORET; ORTEGA, 2014), de modo que tal integração seja produtiva a fim de informar ambas as áreas de investigação - de ensino de línguas embasado em tarefas (task-based language teaching [TBLT], ELLIS, 2003; VAN DEN BRANDEN; BYGATE; NORRIS, 2009) e de aprendizagem de línguas assistida por computador (computer-assisted language learning [CALL], CHAPELLE, 2009).

Neste sentido, a implementação de tarefas com histórias digitais (HDs), a fim de otimizar a produção oral na sala de aula de línguas, aparece como uma alternativa com potencial para preencher essa lacuna - e é esta a proposta da presente pesquisa. Em outras palavras, a investigação teve como objetivo responder a seguinte pergunta de pesquisa: Existe mudança na produção oral em L2 dos participantes como produto do ciclo de tarefas com histórias digitais, considerando-se as dimensões de complexidade, acurácia, fluência, e densidade lexical? Considerando-se o que fora apresentado, a seguinte seção expõe alguns construtos teóricos e estudos relevantes relacionados à questão de produção oral em L2, abordagem de tarefas e histórias digitais, os quais guiaram a proposta de pesquisa posteriormente relatada.

\section{Fundamentação teórica}

Um dos maiores desafios para muitos aprendizes de línguas parece ser, de modo geral, desenvolver a fala na L2, especialmente em um nível mais elevado de proficiência. Segundo Bergsleithner (2009, p. 114) a fala é "uma das habilidades lingüísticas mais difíceis de ser tratada em sala de aula", em especial "quando se trabalha com grupos grandes e/ou muito heterogêneos". Tal questão pode ser explicada através de um viés cognitivo: a ação de produzir língua oralmente é entendida como uma habilidade cognitiva complexa (LEVELT, 1989). Tal habilidade requer prática ${ }^{4}$ a fim de ser desenvolvida de forma plena, já que é por meio da prática que uma habilidade complexa consegue evoluir, passando de um processo inicial controlado a um processo automatizado (SCHMIDT, 1990). Neste sentido, praticar a língua - usá-la em contexto,

\footnotetext{
4 Entende-se aqui o termo prática dentro da perspectiva teórica do processamento da informação, seguindo Schmidt (1990) e Anderson (1995), dentre outros, e não no sentido sugerido por algumas teorias em SLA que entendem prática como a oportunidade de praticar regras específicas por meio de exercícios estruturais ou drills (como em Ellis (1994), por exemplo) (WEISSHEIMER, 2007).
} 
com ênfase maior ao significado da mensagem a ser produzida - é um movimento essencial no processo de aprendizagem de L2. Isso porque, ao produzir a língua, o aprendiz é estimulado a perceber gaps linguísticos (noticing; SCHMIDT, 1990) entre o que consegue dizer e o que quer dizer, a testar suas hipóteses (experimentar, ver como a língua funciona), e refletir sobre a língua em uso (SWAIN, 1993, 1995). Estes aspectos evidenciam resultados da Hipótese do Output, proposta por Swain (1993), a qual tem sido investigada tanto nas áreas de aquisição de L2 (second language acquisition, SLA) quanto de TBLT $^{5}$.

A abordagem de ensino nomeada TBLT, segue uma perspectiva que enfatiza um trabalho pedagógico centrado no aluno, com individualização instrucional, com aprender fazendo (learn by doing) possibilitando a este desenvolver autonomia (VAN DEN BRANDEN; BYGATE; NORRIS, 2009). Além disso, potencializa um aprendizado voltado à l'educacion integrale, segundo Long (2015), entendendo a importância de educar o indivíduo por completo. Por ter uma base teórica solidamente aliada a evidências de pesquisas recentes na área de psicologia cognitiva (em especial de processamento da informação), esta abordagem busca, desse modo, fomentar o aprendizado da L2 por meio de tarefas que demandam uso da língua em contexto real, autêntico, com foco primordial no significado, na construção de sentidos - apesar de garantir espaço secundário para foco na forma ${ }^{6}$ (LONG, 1991), ou seja, para desenvolver aspectos formais da língua também essenciais ao processo de aprendizagem (ELLIS, 2003). Apesar do trabalho pedagógico ser centrado no aluno, o papel do professor é fundamental, uma vez que atua tanto como mediador do processo de aprendizagem, quanto como pesquisador, ao delinear e implementar tarefas na sala de aula, seguindo parâmetros da abordagem, e colaborando assim com o avanço da TBLT como área consolidada de pesquisa em SLA (VAN DEN BRANDEN, 2016).

Por serem entendidas como atividades de sala de aula destinadas a desenvolver a aprendizagem de línguas, por meio das quais os alunos usam a língua, na forma oral ou escrita, com foco no significado (BYGATE, 2015), as tarefas podem ser facilmente conectadas com tecnologias digitais, que também fazem parte do mundo real do aprendiz de L2. Assim, uma conexão possível é a história digital (HD) - uma história curta e envolvente em formato de vídeo, criada a partir da combinação de diferentes

\footnotetext{
De modo geral, grande parte das pesquisas que seguem a abordagem TBLT tendem a analisar a produção (oral ou escrita) em L2 por meio de três dimensões principais - complexidade, acurácia e fluência (ou CAF) - apesar de não haver ainda um consenso na área sobre como definir e operacionalizar de cada dimensão (veja Craven (2017) e Housen e Kuiken (2009) sobre características e limitações de CAF). Recentemente, mais pesquisas têm buscado aliar essas medidas 'tradicionais' a outras medidas, como a denominada adequação comunicativa (adequacy), proposta por Pallotti (2009), por exemplo (veja Specht (2017) para uma discussão do por que usar a medida de adequação para investigar performance). De certa forma, tenta-se assim trazer um viés mais holístico para a investigação da produção oral em L2 no ensino embasado em tarefas. Nossa pesquisa, por exemplo, que resulta no presente artigo, analisou as produções orais dos alunos fazendo uso de tanto de medidas de CAF, além de densidade lexical, quanto de adequação comunicativa, seguindo Pallotti (2009) e Specht (2017). Mais informações quanto a esses elementos são apresentadas na Metodologia.

6 Para Long, o foco na forma, (focus on form) pode auxiliar os aprendizes a perceber gaps no input (SCHMIDT, 1990, 2001) que podem não ser percebidos de outra forma. Ao notar tais lacunas na fala, a produção oral na L2 pode ser otimizada, segundo Swain (1993, 1995).
} 
modalidades, tais como imagem, música, texto, e narrativa oral (CHRISTIANSEN; KOELZER, 2016).

As HDs têm sido amplamente investigadas nas áreas de Educação e de ensino de línguas (LAMBERT, 2007; LEE, 2014; ROBIN; MCNEIL, 2012; SADIK, 2008; SMEDA; DARICH; SHARDA, 2013). De modo geral, as pesquisas em L2 indicam que, ao construir uma HD, o aprendiz pode melhor desenvolver questões formais da língua, além de outras habilidades como, por exemplo, a digital, de pensamento crítico, de trabalho colaborativo, interpessoal, dentre outras (NISHIOKA, 2016; YUSKEL; ROBIN; MCNEIL, 2011). Pesquisas com HDs voltadas para a questão do desenvolvimento da oralidade em L2 tem sido majoritariamente de cunho qualitativo, refletindo, em geral, relatos de aprendizes que percebem melhora no desempenho oral depois de fazer tal atividade. Apesar de pesquisas com esse viés serem extremamente importantes para compreendermos o potencial de HDs para o desenvolvimento dessa habilidade linguística, pouco se sabe se tal percepção (a dos alunos, ao se autoavaliarem) equivale a uma mudança/melhora concreta da fala em L2, por exemplo. São poucos os estudos quantitativos que buscam mensurar ${ }^{7}$ os efeitos do uso de HDs na produção oral em L2 - olhando para gravações orais dos alunos, por exemplo, e avaliando estatisticamente variáveis da dimensão da fala em momentos diferentes, antes e depois de uma intervenção com HD. Nenhum estudo no Brasil com tal enfoque fora identificado até o momento, por exemplo, o que justifica a presente pesquisa, especialmente atentando-se para a importância de que tal proposta sirva para melhor esclarecer as questões anteriormente elencadas, trazendo assim implicações pedagógicas relevantes ao contexto brasileiro. Na sequência, serão apresentados aspectos relativos a como a pesquisa foi organizada e conduzida.

\section{Metodologia}

A presente pesquisa, de cunho quantitativo ${ }^{8}$, buscou investigar o efeito da criação de HDs na produção oral em L2. Para guiar a investigação, a seguinte pergunta de pesquisa foi gerada:

Existe mudança na produção oral em L2 dos participantes como produto do ciclo de tarefas com histórias digitais, considerando-se as dimensões de complexidade, acurácia, fluência, e densidade lexical?

\footnotetext{
Não se quer aqui afirmar que um certo tipo de pesquisa seja mais importante que outro; entende-se, pelo contrário, que ambas - pesquisas de natureza qualitativa e quantitativa - são essenciais e deveriam, quando possível, ser complementares na discussão de qualquer fenômeno linguístico, já que podem amplificar o olhar do pesquisador para seu objeto de estudo. E é exatamente por esse motivo que nossa pesquisa, relatada neste artigo, é de natureza quali e quanti, integrando as duas perspectivas para melhor discutir e analisar os dados.

8 Como já mencionado, este artigo é um fragmento da tese de doutorado da primeira autora, feita sob orientação da segunda autora; assim, discussões mais gerais relacionadas à parte quantitativa da pesquisa serão aqui apresentadas. Contudo, o estudo analisou também, qualitativamente: a) os tipos de processos (meta)cognitivos nos quais os alunos se engajaram ao executar as tarefas do ciclo; e b) as percepções dos mesmos acerca da experiência de construir uma HD em inglês, como um todo. Para mais informações, veja Trevisol (2019).
} 
A fim de responder tal questão, serão apresentados, na sequência, informações acerca dos participantes da pesquisa, dos instrumentos utilizados e dos procedimentos empregados para a coleta dos dados.

\section{Participantes e contexto da pesquisa}

Os participantes da pesquisa foram quatorze aprendizes de inglês -7 homens e 7 mulheres, com idades entre 18-50 anos -, todos licenciandos do curso de Letras Língua Inglesa e Literaturas de uma universidade pública do estado da Bahia. Todos residiam em cidades localizadas no interior do estado da Bahia. Os aprendizes faziam parte de uma turma fechada do curso, estando todos regularmente matriculados nas disciplinas de Intermediário I e Produção Oral I quando os dados foram coletados. Todos aceitaram participar voluntariamente da pesquisa ${ }^{9}$. Com relação à proficiência, 7 participantes foram avaliados como sendo de nível básico e 7 de nível intermediário ${ }^{10}$. Os instrumentos e procedimentos de coleta e análise dos dados serão apresentados a seguir.

\section{Instrumentos, procedimentos de coleta e análise de dados}

A fim de operacionalizar o estudo, alguns dos instrumentos ${ }^{11}$ utilizados para a coleta dos dados foram: a) um pré-teste de produção oral, feito antes do início do ciclo de tarefas; b) um ciclo de tarefas com HD e outras atividades pedagógicas; c) um pós-teste 1 de produção oral, imediato, feito logo após a conclusão do ciclo de tarefas; e d) um pós-teste 2 de produção oral, posterior, feito um mês após a finalização do experimento da criação da HD em inglês. Todos esses instrumentos e tarefas foram desenvolvidas durante três semanas de atividades: os encontros ocorreram 2 vezes por semana, às quartas e quintas-feiras no período noturno (4 horas de duração a cada dia), e em um total de 6 aulas (cerca de 20 horas em sala, aproximadamente), tempo este disponibilizado pelos professores das duas disciplinas mencionadas (os quais não participaram da pesquisa) no mês e novembro de 2017. Todas as atividades da pesquisa foram aplicadas pelo pesquisador, que não havia tido nenhum tipo de contato com os participantes antes do início da coleta de dados.

Além dos instrumentos especificados, os participantes também responderam a sete questionários que auxiliaram na parte qualitativa da pesquisa, possibilitando, por exemplo, o entendimento e discussão de processos cognitivos nos quais os aprendizes se engajaram ao executar cada uma das tarefas do ciclo, e das percepções dos mesmos

\footnotetext{
Este estudo foi aprovado pelo Comitê de Ética em Pesquisa (Parecer 84509118.7.0000.0121).

A proficiência foi medida por sete avaliadores da área de ensino de L2 quando da análise dos dados.

11 Todos os participantes responderam a um termo de consentimento livre e esclarecido antes de iniciar as atividades, atestando seu aceite em participar da pesquisa.
} 
sobre a criação de uma histórias pessoal, do uso de tecnologia digital dentro e fora da sala de aula de línguas, dentre outras questões também relevantes ${ }^{12}$.

Considerando-se o ciclo de tarefas, as atividades destacadas foram: 1) uma gravação oral inicial para contextualizar a temática da HD - minha trajetória como aprendiz de línguas; 2) a escrita do script da HD seguindo a mesma temática; 3 ) a organização do Storyboard, com seleção de imagens e trilha sonora; 4) a gravação do áudio da HD para incluir no vídeo; 5) a finalização da HD, com ajustes finais e sincronização de sons e imagens. Todas as tarefas do ciclo foram produzidas pelos aprendizes em inglês. $\mathrm{O}$ ciclo também incluiu, dentre outras atividades, uma oficina teórico-prática sobre o software Moviemaker para que os participantes pudessem usá-lo na criação da HD, uma vez que a grande maioria não tinha experiência alguma em manipulação de softwares para edição de vídeos.

Dados de três produções orais (PO) individuais em inglês foram coletados, com o uso do whatsapp usando os celulares dos próprios alunos, em diferentes momentos do estudo (ver instrumentos citados nos itens b, d, e). As POs eram narrativas orais individuais curtas (cerca de 60 segundos), em língua inglesa, realizadas após 10 minutos de planejamento não-guiado, ou seja, sem auxílio do professor. Todas as POs seguiam os mesmos procedimentos. Ao planejar, os alunos podiam tomar notas sobre o que iriam posteriormente falar; porém, após o término do tempo estipulado para o planejamento, a gravação individual era feita sem o auxílio das anotações escritas. As POs e demais atividades do ciclo foram realizadas em uma sala de aula da universidade na qual os participantes frequentavam. O tema das POs e da HD era o mesmo, sendo um tópico familiar a todos os alunos: estimulava-os a pensar e contar sobre suas trajetórias enquanto aprendizes de inglês. Assim como as POs, a HD foi também produzida individualmente em língua inglesa - as narrativas produzidas em formato digital tinham um mínimo de 2 e máximo de 5 minutos de duração.

Quanto à operacionalização das variáveis de fala na L2, após coletadas e transcritas em sua totalidade, as POs foram medidas seguindo a tradição das pesquisas na área de tarefas (SKEHAN, 2009b), que em geral considera as seguintes dimensões: complexidade, acurácia, fluência (aqui avaliada por meio de seis medidas específicas) e densidade lexical. Em termos gerais, a complexidade foi operacionalizada ${ }^{13}$ considerando-se o número de orações subordinadas por AS-unit ${ }^{14}$. A acurácia foi operacionalizada

12 Considerando-se que a discussão da parte qualitativa do estudo está fora do escopo desse artigo, maiores informações podem ser encontradas em Trevisol (2019), Trevisol e D’Ely (2019) e Delatorre e Trevisol (2020). Além disso, uma descrição detalhada do questionário inicial do estudo pode ser acessada em Trevisol (2020).

13 A fim de operacionalizar tais medidas, alguns recursos tecnológicos foram utilizados na fase de análise de dados, tais como o Microsoft Word (para organizar documentos, como a transcrição das falas), o Microsoft Excel (para organizar toda a parte numérica em planilhas, tabelas, com uso de funções para calcular médias), os software Audacity e Praat (para verificar tempo de fala e calcular medidas relacionadas à fluência), o Text Analyzer (https://www.online-utility. org/text/analyzer.jsp) para calcular a frequência das palavras e medir densidade lexical), e, por fim, o software R (para as análises estatísticas). Mais informações quanto aos procedimentos operacionais e de análise podem ser encontradas em Trevisol (2019).

14 AS-unit (analysis of speech unit) é uma unidade de análise de fala, sendo uma medida padrão mais refinada para investigações relacionadas à fala. Proposta por Foster, Tonkyn e Wigglesworth (2000), a AS-unit tem sido amplamente 
pelo número de erros por AS-unit - erros entendidos aqui como desvios da norma padrão. Já a fluência foi operacionalizada, considerando-se ${ }^{15}$ as seguintes medidas: a) a velocidade de fala, operacionalizada pelo número de palavras produzidas por minuto medidas denominadas pruned $^{16}$ e unpruned; b) quebra de fluência ou disfluência, operacionalizada pelo número de pausas (filled e unfilled $^{17}$ ) por AS-unit, por tempo de pausas com silêncio (unfilled pauses), medido em porcentagem; e c) e reformulações, operacionalizada pelo número de auto-correções (self-repairs) por AS-unit. Por fim, a densidade lexical ponderada foi calculada, considerando-se a proporção de itens lexicais e sua frequência. Os dados orais foram todos analisados posteriormente por meio de estatística descritiva (e.g., cálculo de médias, desvio padrão, valores mínimos e máximos, score de ganhos, dentre outros) e inferencial (e.g., testes estatísticos de normalidade da amostra, de comparação entre os momentos do experimento (e.g., Friedman), dentre outros).

Vale mencionar ainda que, aos interessados em desenvolver estudos considerando as dimensões empregadas na presente pesquisa - dimensões aqui brevemente descritas -, informações mais detalhadas sobre cada uma das medidas e sobre como foram operacionalizadas são apresentadas em Trevisol (2019) de modo bastante didático. Além disso, as transcrições de todas as POs estão apresentadas nos anexos do referido trabalho de forma ilustrativa, a fim de possibilitar que outros pesquisadores consigam melhor compreender como se deu a organização geral e de análise dos dados ${ }^{18}$, facilitando, desse modo, que estudos similares sobre produção oral em L2 possam ser realizados tanto dentro quanto fora do Brasil.

\section{Resultados}

Ao fim do experimento, que culminou na criação individual de uma HD em inglês, percebeu-se que as POs em L2 de todos os participantes apresentaram mudança positiva em algum dos elementos investigados. Em outras palavras, as falas em inglês dos

utilizada em estudos recentes de produção oral em L2, tais como Ahmadian, Tavakoli e Dastjerdi (2015), Norris e Ortega (2009), Révész, Ekiert e Torgersen (2014) e Specht (2017), dentre outros.

15 Seguindo Skehan (2003), a fluência é geralmente entendida como uma dimensão que se subdivide em velocidade da fala, quebra da fala (e.g., pausa) e reformulações produzidas pelo falante.

16 Na medida pruned são desconsideradas as repetições e reformulações, por exemplo (e.g., quando o aluno erra e se auto-corrige logo em seguida), enquanto que na unpruned esses elementos são mantidos para o cálculo final da velocidade da fala produzida.

17 Filled pauses ou pausas preenchidas são aquelas nas quais o falante produz itens como humm, ehh, well, de modo estratégico, a fim de ter tempo para pensar no que irá dizer na sequência. Unfilled pauses, por outro lado, são pausas de completo silêncio.

18 A disponibilização de tais elementos nos anexos permite também que os dados da pesquisa possam ser verificados, questionados, e discutidos criticamente por outros pesquisadores da área. Além disso, àqueles ainda não familiarizados com pesquisa na área de TBLT, em especial pesquisa com enfoque na oralidade em L2, os anexos servem como suporte para que esse contato inicial aconteça, guiando futuros pesquisadores no passo-a-passo instrumental de mensurar as dimensões de complexidade, acurácia, fluência e densidade lexical. 
participantes depois do experimento com HDs foram melhores, em geral, do que suas produções de antes da atividade com a HD ser desenvolvida - apesar de tal diferença entre antes e depois ter sido, em termos numéricos, pequena.

De modo a ilustrar de forma simples tais mudanças, a Tabela 1, a seguir, apresenta os ganhos individuais de cada participante nos dois pós-testes. Tais ganhos foram calculados em comparação ao momento inicial do experimento - o pré-teste - feito antes de terem início as atividades para construção da HD em sala de aula. $\mathrm{O}$ cálculo foi feito subtraindo-se do valor da medida do pós-teste (1 ou 2) o valor da medida no pré-teste inicial, observando, portanto, a diferença entre o valor do pós e o valor do pré-teste. $\mathrm{Na}$ tabela, as dimensões de fala aparecem nas colunas, considerando-se o total de nove medidas analisadas - de complexidade (medida 1), a densidade lexical (medida 9). O símbolo mais (+) revela em qual medida de fala em L2 houve ganho, ou melhora, para cada um dos participantes. As POs estão divididas na tabela em PO3 pós-teste 1 , imediato - e PO4 - pós-teste 2 -, feito cerca de um mês após a finalização das atividades com HD. Além disso, na coluna de Participantes, as linhas iniciais - P1, P7, P9, P10, P11, P12 e P13 - referem-se aos participantes avaliados como sendo de nível básico; enquanto que os participantes na sequência - P2, P3, P4, P5, P6, P8, e P14 - são os de nível intermediário. 
Tabela 1 - Ganhos individuais em produção de fala na L2: resultados do pós-teste

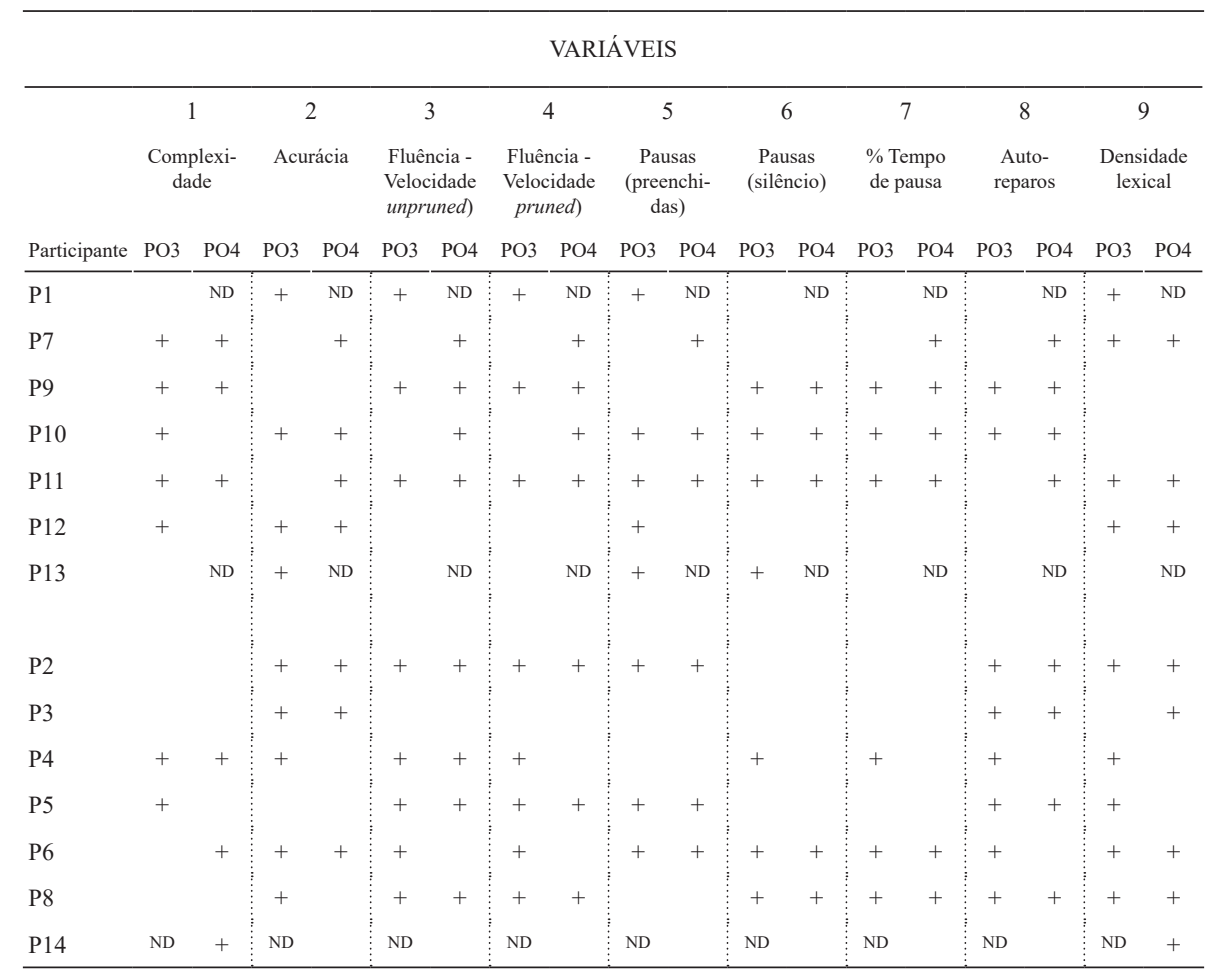

Nota: P refere-se à Participante; ND (no data) refere-se aos valores ausentes, não coletados; PO3 refere-se ao pós-teste 1 (imediato) e PO4 ao pós-teste 2 (posterior); o símbolo + refere-se a ganho, observação positiva ou instância na qual foi observada melhora na fala do participante.

Fonte: Elaboração própria.

De modo geral, a tabela evidencia que, para este grupo de aprendizes, as produções orais em língua inglesa feitas após o ciclo de tarefas com histórias digitais apresentaram melhora em vários aspectos, apesar de não ser possível perceber um padrão de modo geral. Alguns participantes apresentaram melhora na fala em L2 em várias medidas (e.g., P6, P11), enquanto que outros em poucas medidas (e.g., P12, P14), por exemplo.

A fim de exemplificar ${ }^{19}$ algumas questões, olhemos para o pós-teste imediato (PO3) do participante 6 (de nível intermediário) especificamente: percebe-se que a produção oral em L2 de P6 foi mais acurada e mais rápida (em ambas as medidas,

19 Na tentativa de facilitar a leitura da Tabela 1, consideremos por exemplo: se o símbolo + aparece em uma dada medida de fala (de 1 a 9) para um dado participante (P) tanto nas colunas de PO3 quanto em PO4, isso significa que houve melhora na produção oral em L2 dessa medida tanto imediatamente ao fim do ciclo de tarefas com HD (PO3) quanto posteriormente, um mês depois de findado o ciclo (PO4). Nesse sentido, a melhora se manteve com o passar do tempo. Se o símbolo + aparece em uma dada medida de fala só em PO3 (e não em PO4), entende-se que houve melhora imediata (ao término do ciclo), porém a mesma não se manteve posteriormente, em PO4. Vejamos, por exemplo, P5 e as medidas de acurácia e densidade lexical: houve melhora (+) em PO3 mas não em PO4. 
pruned e unpruned); além disso, sua fala teve menos pausas em geral (e.g., menos elementos como humm e menos silêncio), menos momentos de auto-correção e o léxico empregado foi mais variado se comparado ao do pré-teste. Assim, a produção oral na L2 de P6 no momento do pós-teste imediato teve ganhos em 8 das 9 variáveis investigadas, sendo que somente para a medida de complexidade nao foi observada nenhum ganho (ou seja, não houve aumento no número de orações subordinadas por AS-unit produzidas em PO3).

Ainda de modo ilustrativo, analisando a produção oral em L2 de P6 um mês após a conclusão das atividades com HDs - olhando agora portanto para PO4, pós-teste 2 -, percebe-se ganho ou melhora na produção agora para a dimensão de complexidade da fala - ou seja, observa-se que a produção oral de P6 é mais complexa no momento $\mathrm{PO}^{20}$ (um mês depois de finalizado o trabalho com HDs) se comparada ao início do mesmo (no pré-teste, realizado antes do ciclo de tarefas com HDs). Ainda analisando a produção de P6: ganhos $(+)$ são também observados na dimensão acurácia (a melhora já percebida em PO3 parece ter sido mantida em PO4); além disso, a quantidade de pausas foi também menor (se manteve menor) e a produção lexical também melhorou em PO4. Assim, a fala em inglês de P6 no pós-teste 2 apresentou melhora em 6 das 9 variáveis, sendo elas relacionadas às dimensões de complexidade, acurácia, fluência em termos de pausas (ou quebra de fluência) - e densidade lexical. Percebe-se portanto, a partir do exemplo descrito, que a presente investigação permitiu um olhar tanto individual, considerando as produções de cada participante, quanto um olhar mais amplo dos grupos, de modo separado, considerando-se as diferenças de proficiência dos aprendizes (e.g., comparando os níveis básico e intermediário). Além disso, a análise evidenciou efeitos positivos na performance em L2 para a turma como um todo - o que é extremamente relevante para pesquisas que, tal como essa, busquem compreender melhor os efeitos de tarefas aliadas à ferramentas de tecnologia digital em salas de aula 'reais' - afinal, aqui, os participantes compõem uma turma fechada, intacta, que reflete um contexto de uma sala de aula entendida como 'real' (e.g., não criada para fins de um único experimento, sem coleta de dados em laboratório, por exemplo).

Considerando-se, agora, os resultados para o grupo de aprendizes como um todo $(\mathrm{N}=14)$, percebeu-se que as POs melhoraram especialmente para as dimensões de acurácia, fluência e densidade lexical, tanto no pós-teste 1 (imediato) quanto no pósteste 2 (posterior, realizado cerca de um mês depois de o experimento ser concluído). Tal avanço se deu, possivelmente, pelo efeito da repetição da tarefa (BYGATE, 2001; D’ELY, 2006; D'ELY; MOTA; BYGATE, 2019), desencadeado tanto pelas atividades das gravações do whatsapp (as 3 POs produzidas) quanto - e especialmente - pela tarefa de gravar a narrativa oral que faria parte da HD. A gravação da narrativa com sua própria voz para a HD (com base no script escrito, construído previamente em sala) foi uma atividade feita pelos participantes em casa; assim, os mesmos puderam ter mais tempo para realizar a atividade, fazendo-a, autonomamente, da forma como

20 Conforme representado pelo símbolo $(+)$ na tabela. 
julgassem mais adequada. Isso possibilitou que cada um pudesse repetir a gravação mais de uma vez, caso julgassem necessário.

Os resultados evidenciam que, dada a oportunidade de realizar a atividade de gravação do áudio da HD fora de sala de aula, muitos participantes realizaram essa tarefa considerando-a como um momento de ensaio (rehearsal) de suas falas. Suas reflexões acerca desse processo ${ }^{21}$ demonstram que eles conseguiram, por meio de tal atividade: a) perceber lacunas ou gaps em sua própria fala - notando diferenças entre o que queriam dizer e o que eram capazes de dizer na gravação, de fato; e b) realizar uma auto-análise de de suas produções orais, com levantamento de pontos críticos e elementos específicos a serem revistos, modificados (e.g., pronúncia, revisão de um dado tempo verbal). Tais questões são entendidas como importantes para a aprendizagem de L2, e especificamente para o desenvolvimento da produção oral, segundo Swain (1993, 1995) e Swain e Lapkin (1995).

Muitas pesquisas interessadas no aprimoramento da oralidade em L2 reforçam a relevância da repetição ${ }^{22}$ de uma mesma tarefa por favorecer o desenvolvimento da PO na L2 (como, por exemplo, BIRJANDI; AHANGARI, 2008; BYGATE, 1996, 1998, 1999, 2001, 2009; BYGATE; SAMUDA, 2005; D’ELY, 2006; D’ELY; MOTA; BYGATE, 2019; FINARDI, 2008), especialmente com relação às dimensões de complexidade, acurácia e/ou fluência (SKEHAN, 2014). Analisando-se individualmente as POs por nível de proficiência, por exemplo, observou-se que, de modo geral, mais POs do grupo intermediário melhoraram no pós-teste imediato (PO3), enquanto que mais POs do grupo básico melhoraram no pós-teste posterior. Tal observação sugere que um tempo maior pode vir a ser necessário para que mudanças - ou algum tipo de efeito do tratamento - sejam efetivamente notadas na performance de aprendizes de L2 em níveis iniciais. Isso se dá, possivelmente, porque a automatização e/ou reestruturação da língua não avança do dia para a noite: para que um processo antes controlado venha a se tornar automatizado, há que se praticar (SCHMIDT, 1990), o que pressupõe tempo e frequência de uso, por exemplo. Tais questões refletem o que acontece para se desenvolver a oralidade - habilidade cognitiva complexa, segundo Levelt (1989) - assim como a proficiência na L2, de modo geral.

Apesar de mudanças positivas terem sido observadas nas falas em L2 dos participantes da pesquisa após a criação das narrativas digitais, o resultado do teste de Friedmam sugere que as diferenças entre os momentos não foram estatisticamente

21 Tais informações foram coletadas por meio dos questionários mencionados de forma breve anteriormente. Por conta disso, a pesquisa conseguiu triangular melhor os dados, contribuindo, assim, para a compreensão sobre várias outras questões pertinentes tanto ao processo de criar uma HD em sala de aula, quanto de aprender lingua (e outras habilidades) por meio dessa tarefa e ferramenta.

22 Entende-se que, ao repetir a tarefa, de modo geral, a carga de processamento cognitivo tende a ser reduzida; isso favorece a produção posterior (repetida), já que a informação previamente ativada na memória pode ser reintegrada na performance subsequente, permitindo assim que parte dos recursos atencionais sejam liberados a outros aspectos da performance (BYGATE, 2001). Desse modo, esse processo metacognitivo como um todo - da repetição da tarefa possibilita certa otimização do desempenho na L2, ao menos em alguma dimensão da fala. 
significativas $^{23}$ (ao nível de 5\% de significância) para nenhuma das nove variáveis investigadas. Tal resultado implica, portanto, na impossibilidade de generalização de tais resultados para a população de aprendizes de L2 como um todo. Esse resultado se deve, possivelmente, ao tamanho da amostra e ao tempo total do experimento com HDs, além de outras questões e limitações da pesquisa, as quais serão expostas na sequência, juntamente com algumas implicações relacionadas à pedagogia de línguas.

\section{Considerações finais}

O presente artigo teve como objetivo relatar uma parte da investigação acerca dos efeitos de um ciclo de tarefas com HDs na produção oral de aprendizes, futuros professores de inglês. $\mathrm{O}$ experimento foi concebido seguindo pressupostos da abordagem de tarefas (SKEHAN, 2009a), buscando aliar as potencialidades das tecnologias digitais à aprendizagem de L2 em contextos educacionais mais heterogêneos, como por exemplo, uma sala de aula 'real'. Buscou-se também na pesquisa, de modo geral (de modo bastante resumido), compreender melhor como se deu o processo de implementação de tarefas com HD, a fim de fomentar o conhecimento acerca de como implementar tarefas com ferramentas digitais, compreender seu possível impacto no aprendizado de L2 - ambas questões que ainda carecem de pesquisas empíricas no contexto brasileiro -, e, por fim, discutir aspectos importantes para a pedagogia de línguas. Como demonstrado, os resultados sugerem que tarefas de criação de HDs podem ser usadas como uma alternativa em potencial para a sala de aula de línguas, uma vez que fomentam o uso efetivo da L2 de modo integrado - já que várias habilidades linguísticas são trabalhadas na HD (e.g., leitura, escrita, fala, compreensão auditiva) -, com possível melhora da produção oral ao final do ciclo, mesmo que em um curto espaço de tempo.

Apesar de os resultados terem sido positivos, em especial para os participantes desse contexto, essa investigação apresenta uma série de limitações, dentre as quais destacam-se, por exemplo: 1) o número reduzido de participantes $(\mathrm{N}=14)$, comum em classes como a do contexto investigado; e 2) o tempo extremamente curto do ciclo de tarefas com HD, considerando-se outras pesquisas da área.

Quanto à 1: Pesquisas na área de TBLT tendem a ser desenvolvidas com um número maior de participantes; entretanto, raramente são conduzidas, considerando-se ciclos de atividades de longo prazo (i.e., experimentos longitudinais) e especialmente em contextos de sala de aula intacta/real, que tende a apresentar maior heterogeneidade (e/ ou fatores que fogem ao controle do pesquisador e que podem vir a afetar os resultados da pesquisa, por exemplo). Entende-se que, em geral, ao se ter mais participantes de pesquisa, maiores são as chances de se obterem resultados com valor de $p$ significativo.

23 Primeiramente, usou-se o teste de Shapiro Wilk para verificação da normalidade da amostra; além disso, rodou-se o teste não-paramétrico de Friedman (para amostras dependentes e pareadas) a fim de verificar a existência de diferenças entre os 3 momentos do experimento (pré-teste e pós-testes 1 e 2). 
Portanto, é possível que, havendo um maior número de aprendizes no grupo, maiores seriam as chances de que resultados estatisticamente significativos fossem obtidos.

Quanto à 2: Pesquisas com HD têm sido desenvolvidas em períodos que variam de três a doze meses em geral. Na investigação de Nishioka (2016), por exemplo, os participantes trabalharam colaborativamente por um semestre inteiro para construir suas HDs; enquanto isso, nesta pesquisa, o ciclo completo de tarefas - que culminou na apresentação individual das HD ao grupo todo de aprendizes, realizado no final do ciclo - foi feito em apenas três semanas ${ }^{24}$ (e o pós-teste posterior em um dia apenas). Por ter sido desenvolvida em uma sala de aula 'real' (não-experimental, heterogênea), a disponibilização de maior tempo para o experimento não foi possível em virtude de vários fatores, dentre eles, a finalização do semestre letivo na universidade.

Levando em conta o que fora apresentado anteriormente, sugere-se que futuras pesquisas e/ou práticas pedagógicas considerem tais questões e busquem, na medida do possível, refletir acerca de como melhor adaptar ciclos de tarefas com HD para os seus contextos em particular, considerando especialmente ciclos mais longos para o desenvolvimento dessa atividade. Além disso, havendo maior tempo disponível, sugere-se o planejamento de atividades adicionais que componham as fases durante ou pós-tarefa ${ }^{25}$ do ciclo (quando os alunos estão iniciando a escrita do texto que servirá de script para a HD ou após terem apresentado o vídeo aos colegas da classe) a fim de melhor atender as necessidades dos aprendizes.

Assim, algumas questões a serem consideradas pelo professor ao implementar um ciclo de tarefas com HDs seriam, por exemplo, relacionadas à: a) auxiliar com a organização da escrita e reescrita do draft da HD, fazendo com que a ação de escrever sobre a temática da história seja acompanhada pelo professor (com feedback) e desenvolvida de modo processual; b) propor atividades de transcrição de gravações de áudio (i.e., do whatsapp) que fomentem momentos de análise conjunta ou individual da fala, com oportunidades para dirigir a atenção a certos elementos da língua (e.g., gramática, dificuldades de pronúncia, dentre outros). Ao se considerar tais questões, promover-se-ia, possivelmente, maior foco na forma, reflexão da língua (do que fora produzido na L2), noticing ou percepção mais atenta de aspectos formais não notados individualmente. Tais elementos implicam na ampliação de oportunidades de desenvolvimento da L2 - em particular da habilidade oral - entendida como complexa e carente de atenção, especialmente no ambiente de aprendizagem-ensino formal.

Por fim, considerando-se que a presente investigação trouxe evidências de alguns efeitos positivos de histórias digitais na produção oral em L2 em uma sala de aula 'real', entende-se que ao aliarmos tecnologias digitais com tarefas pedagógicas cuidadosamente pensadas, planejadas e implementadas pelo professor - maiores parecem ser as chances de que o aprendiz de L2 assuma seu papel no centro do processo

\footnotetext{
24 Além do pouco tempo, convém destacar que os aprendizes foram ainda desafiados a desenvolver uma nova habilidade digital durante a pesquisa: aprender a usar o software de edição de vídeo - Moviemaker - novidade para a maioria dos participantes e essencial para a criação da HD.

25 As fases de implementação de tarefas seguem o framework de Skehan (2009a).
} 
de aprendizagem, conseguindo, desse modo, melhor desenvolver as competências que lhe cabem. Mais pesquisas, porém, se fazem necessárias a fim de que consigamos aprofundar o conhecimento acerca dos vários fatores que podem influenciar esse processo, em especial ao integrarmos tecnologia em ambientes de aprendizagem formais de língua, ambientes estes por vezes com características tão locais e específicas, ansiando por serem melhor conhecidos e socializados.

TREVISOL, J. R.; D'ELY, R. C. F. Effects of implementing digital storytelling on the oral production of English learners: a task-based study. Alfa, São Paulo, v. 65, 2021.

- ABSTRACT: Considering the rapid change of technology nowadays and the possibilities of using new digital tools for building knowledge in different contexts, the interest of researchers and practitioners has grown considering how to best allign digital technologies and education. Nonetheless, plenty of unanswered doubts remain regarding the more adequate ways in which technology and pedagogical activities can be integrated as a potential for learning, especially in second language (L2) contexts. Taking this into consideration, the present study aimed at investigating the effects of a task cycle (ELLIS, 2003) with digital storytelling on the oral production of L2 learners of English at a public university of Bahia. Oral data from fourteen participants were gathered in three moments - pre and post-tests-via Whatsapp and analysed considering complexity, accuracy, fluency and lexical density dimensions (SKEHAN, 2003). Results in general suggest an improvement in participants' 22 speech for some of the measures investigated, despite the short experimental time. Therefore, the potential of digital storytelling is discussed as an alternative tool for L2 enhancement in formal teaching and learning contexts, taking into account the need for further studies whose aim is to unveil and inform issues about the use of technologies in the language classroom.

- KEYWORDS: Digital technology. Digital Storytelling. Tasks. Oral production. Language learning and teaching. Classroom.

\section{REFERÊNCIAS}

AHMADIAN, M. J., TAVAKOLI, M.; DASTJERDI, H. The combined effects of online planning and task structure on complexity, accuracy, and fluency of L2 speech. Language Learning Journal, Ann Arbor, v. 43, n. 1, p. 41-56, 2015.

ANDERSON, J.R. Learning and memory: an integrated approach. New York: John Wiley and Sons, 1995.

APPEL, C.; BORGES, F. Task design for L2 oral practice in audioblogs. The EUROCALL Review, Valencia, v. 20, n. 1, 2011. 
BERGSLEITHNER, J. M. Linguagem oral e aspectos cognitivos em Linguística Aplicada: ensino/aprendizagem de L2/LE através de tarefas. Revista Lingua \& Literatura, Frederico Westphalen, v. 11, n. 17, p. 113-124, 2009.

BIRJANDI, P.; AHANGARI, S. Effects of Task Repetition on the Fluency, Complexity and Accuracy of Iranian EFL Learners. The Asian EFL Journal, Saudi Arabia, v. 10, n. 3, p. 28-52, 2008.

BUCKINGHAM, D. Media education goes digital: an introduction. Learning, Media and Technology, London, v. 32, n. 2, p. 111-119, 2007.

BYGATE, M. Introduction. In: BYGATE, M. (ed.). Domains and directions in the development of TBLT. Amsterdam: John Benjamins, 2015. p.XV-XXIV.

BYGATE, M. Effects of task repetition on the structure and control of oral language. In: VAN DEN BRANDEN, K.; M. BYGATE, M.; NORRIS, J. (ed.). Task-based language teaching: a reader. Philadelphia, PA: John Benjamins, 2009. p. 249-274.

BYGATE, M. Effects of task repetition on the structure and control of oral language. In: BYGATE, M.; SKEHAN, P.; SWAIN, M. (ed.). Researching pedagogic tasks: second language learning and testing. London: Longman, 2001. p.23-48.

BYGATE, M. Task as context for the framing, reframing and unframing of language. System, Amsterdam, v. 27, p. 33-48, 1999.

BYGATE, M. Theoretical perspectives on speaking. Annual Review of Applied Linguistics, Cambridge, v. 18, p. 20-42, 1998.

BYGATE, M. Effects of task repetition: appraising the developing language of learners. In: WILLIS, J.; WILLIS, D. (ed.). Challenge and Change in Language Teaching. Oxford: Heinemann, 1996. p. 136-146.

BYGATE, M.; SAMUDA, V. Integrative planning through the use of task repetition. In: ELLIS, R. (ed.). Planning and task performance in a second language. Amsterdam: New York: Benjamins, 2005. p.37-74.

CHAPELLE, C. A. The relationship between second language acquisition theory and computer-assisted language learning. The Modern Language Journal, Hoboken, v.93, p. 741-753, 2009.

CHRISTIANSEN, M.S.; KOELZER, M.L. Digital storytelling: using different technologies for EFL. MEXTESOL Journal, Ciudad del Mexico, v. 40, n.1, p. 1-14, 2016.

CRAVEN, L. Measuring language performance: complexity, accuracy and fluency measures. In: WEI INTERNATIONAL ACADEMIC CONFERENCE, Barcelona, 2017. Proceedings [...], Barcelona: Spain, 2017. p.25-27. 
DELATORRE, F.; TREVISOL, J.R. Brazilian learners of English perceptions about oral production on a digital storytelling task cycle. Organon, Porto Alegre, v. 35, n. 68, 2020. Disponível em: https://seer.ufrgs.br/organon/article/view/100710. Acesso em: 12 maio 2021.

D'ELY, R. C. F. A focus on learners' metacognitive processes: the impact of strategic planning, repetition, strategic planning plus repetition, and strategic planning for repetition on $\mathbf{L} 2$ oral performance. Tese (Doutorado em Letras) - Centro de Comunicação e Expressão, Universidade Federal de Santa Catarina, Florianópolis, 2006. Disponível em: http://repositorio.ufsc.br/xmlui/handle/123456789/89407. Acesso em: 12 maio 2021.

D'ELY, R. C. F.; MOTA, M.; BYGATE, M. Strategic planning and repetition as metacognitive processes in task performance: implications for EFL learners' speech production. In: ZHISHENG, E.; AHMADIAN, M. J. (ed.). Researching L2 Taskperformance and pedagogy: in honour of Peter Skehan. Amsterdam: John Benjamins, 2019. p. 199-228.

ELLIS, R. Task-based language learning and teaching. Oxford: Oxford University Press, 2003.

ELLIS, R. The study of second language acquisition. Oxford: Oxford University Press, 1994.

EVANS, M. Foreign language learning with digital technology. New York: Continuum International Publishing, 2009.

FINARDI, K. Effects of task repetition on L2 oral performance. Trabalhos em Linguistica Aplicada, Campinas, v. 47, n. 1, p. 31-43, 2008.

FOSTER, P.; TONKYN, A.; WIGGLESWORTH, G. Measuring spoken language: a unit for all reasons. Applied Linguistics, Oxford, v. 21, n. 3, p. 354-375, 2000.

GONZÁLEZ-LLORET, M; ORTEGA, L. Technology-mediated TBLT: researching technology and tasks. Amsterdam: John Benjamins, 2014.

HOUSEN, A.; KUIKEN, F. Complexity, accuracy and fluency in second language acquisition. Applied Linguistics, Oxford,v. 30, n. 4, p. 461-473, 2009.

LAMBERT, J. Digital storytelling cookbook. Berkely, CA: Digital Dinner Press, 2007.

LEE, L. Digital news stories: Building language learners' content knowledge and speaking skills. Foreign Language Annals, Hoboken, v. 47, n. 2, p. 338-356, 2014.

LEVELT, W. J. M. Speaking: from intention to articulation. Cambridge, Massachusets: The MIT Press, 1989.

LONG, M. H. Building the road as we travel. In: BYGATE, M. (ed.). Domains and directions in the development of TBLT. Amsterdam: John Benjamins, 2015. p. 1-26. 
LONG, M. H. Focus on form: a design feature in language teaching methodology. In: DE BOT, K.; GINSBERG, R.; KRAMSCH, C. (ed.). Foreign language research in cross-cultural perspective. Amsterdam: John Benjamins, 1991. p.39-52.

NISHIOKA, H. Analysing language development in a collaborative digital storytelling project: Sociocultural perspectives. System, Amsterdam, v.62, p. 39-52, 2016.

NORRIS, J. M.; ORTEGA, L. Towards an organic approach to investigating CAF in instructed SLA: The case of complexity. Applied Linguistics, Oxford, v. 30, n. 4, p. 555-578, 2009.

PALlOTTI, G. CAF: Defining, refining and differentiating constructs. Applied Linguistics, Oxford, v. 30, p. 590-601, 2009.

RÉVÉSZ, A.; EKIERT, M.; TORGERSEN, E. N. The Effects of Complexity, Accuracy, and Fluency on Communicative Adequacy in Oral Task Performance. Applied Linguistics, Oxford, p. 1-22, 2014.

ROBIN, B. R.; MC NEIL, S. What Educators Should Know about Teaching Digital Storytelling. Digital Education Review, Barcelona, v.22, p. 37-52, 2012.

SADIK, A. Digital storytelling: a meaningful technology-integrated approach for engaged student learning. Education Technology Research Development, Berlin, v. 56, p. 487-506, 2008.

SCHMIDT, R. W. Attention. In: ROBINSON, P. Cognition and second language instruction. Cambridge: Cambridge University Press, 2001. p. 3-32.

SCHMIDT, R. W. The role of consciousness in second language learning. Applied Linguistics, Oxford, v.11, n. 2, p. 17-46, 1990.

SKEHAN, P. The context for researching a processing perspective on task performance. In: SKEHAN, P. (ed.). Processing perspectives on task performance. Amsterdam: John Benjamins, 2014. p. 1-26.

SKEHAN, P. A framework for the implementation of task-based instruction. In: VAN DEN BRANDEN, K.; M. BYGATE, M.; NORRIS, J. (ed.). Task-based language teaching: a reader. Philadelphia, PA: John Benjamins, 2009a. p. 83-108.

SKEHAN, P. Modelling second language performance: Integrating complexity, accuracy, fluency and lexis. Applied Linguistics, Oxford, v. 30, p. 510-532, 2009b.

SKEHAN, P. Task-based instruction. Cambridge: Cambridge University Press, 2003.

SMEDA, N.; DARICH, E; SHARDA, N. The Effectiveness of Digital Storytelling in the Classrooms: A Case Study. In: IEEE INTERNATIONAL CONFERENCE ON ADVANCED LEARNING TECHNOLOGIES, 13., Proceedings [...], 2013. p. 491-492. 
SPECHT, A. L. Is strategic planning enough?: Investigating the impact of two types of strategy instruction on students' oral planned performance. 2017. Dissertação (Mestrado em Inglês) - Centro de Comunicação e Expressão, Universidade Federal de Santa Catarina, Florianópolis, 2017. Disponível em: https://repositorio.ufsc.br/ handle/123456789/187086. Acesso em: 12 maio 2021.

SWAIN, M. Three functions of output in second language learning. In: COOK, G.; SEIDHOFER, B. (ed.). Principles and practice in applied linguistics. Oxford: Oxford University Press, 1995. p.125-144.

SWAIN, M. The output hypothesis: just speaking and writing isn't enough. The Canadian Modern Language Review, Toronto, v. 50, p. 158-164, 1993.

SWAIN, M.; LAPKIN, S. Problems in output and the cognitive processes they generate: A step towards second language learning. Applied Linguistics, Oxford, v. 16, p. 371-391, 1995.

TREVISOL, J. R. A needs analysis questionnaire for the L2 classroom: insights from a Brazilian study on tasks and technology. Revista X, Curitiba, v. 15, n. 3, p. 126-145, 2020.

TREVISOL, J. R. Investigating L2 learners production and perception of a cycle of tasks with digital storytelling: an exploratory study in technology-mediated TBLT. 2019. Tese (Doutorado em Inglês) - Centro de Comunicação e Expressão, Universidade Federal de Santa Catarina, Florianópolis, 2019. Disponível em: https://repositorio.ufsc. br/handle/123456789/198996. Acesso em: 12 maio 2021.

TREVISOL, J. R.; D’ELY, R. C. S. F. Tarefas \& histórias digitais na sala de aula de línguas: efeitos na produção oral em L2. In: OLIVEIRA, D. A.; SILVA, I. T. (org.). Fundamentos e práticas no ensino da língua inglesa: Volume I: Resultados e propostas de pesquisa. Alagoinhas: Bordô-Grená, 2019. p. 79-92. Disponível em: https://5fd55af0-05d2-4627-9691-0c7f536817eb.filesusr.com/ugd/d0c995_ea6fcb10 eeaf4061845932936d784bda.pdf. Acesso em: 12 maio 2021.

TUMOLO, C. H. S. Ensino à distância: horizontes para o ensino de línguas estrangeiras. Fragmentos, Goiânia, v.30, p. 25-34, 2006.

TUMOLO, C. H. S. Histórias digitais como recurso para ensino/aprendizagem de Inglês como língua estrangeira. Estudos Anglo Americanos, Florianópolis, v. 43, p. 100-117, 2015.

VAN DEN BRANDEN, K. The role of teachers in task-based language education. Annual Review of Applied Linguistics, Cambridge, v. 36, p. 164-181, 2016.

VAN DEN BRANDEN, K.; BYGATE, M; NORRIS, J.M. Task-based language teaching: a reader. Amsterdam: John Benjamins, 2009. 
WANG, S.; WÁSQUEZ, C. Web 2.0 and second language learning: what does research tell us? CALICO Journal, Sheffield, v. 29, n. 3, p. 412-430, 2012.

WEISSHEIMER, J. Working memory capacity and the development of L2 speech production. 2007. Tese (Doutorado em Inglês) - Centro de Comunicação e Expressão, Universidade Federal de Santa Catarina, Florianópolis, 2007.

WEISSHEIMER, J.; CALDAS, V.; MARQUES, F. Using whatsapp to develop L2 oral production. Leitura, Maceió, v.1, n. 60, p. 21-38, 2018.

YUSKEL, P.; ROBIN, B.; MCNEIL, S. Educational Uses of Digital Storytelling all around the World. In: SOCIETY FOR INFORMATION TECHNOLOGY \& TEACHER EDUCATION INTERNATIONAL CONFERENCE, 2011, Nashville. Proceedings [...], Nashville: AACE, 2011. p. 1264-1271.

Recebido em 3 de junho de 2019

Aprovado em 4 de junho de 2020 